\title{
Simulations of grafted polymers in a good solvent
}

\author{
Peter Grassberger \\ John-von-Neumann Institute for Computing, Forschungszentrum Jülich, D-52425 Jülich, Germany
}

(Dated: September 6, 2018)

\begin{abstract}
We present improved simulations of three-dimensional self avoiding walks with one end attached to an impenetrable surface on the simple cubic lattice. This surface can either be a-thermal, having thus only an entropic effect, or attractive. In the latter case we concentrate on the adsorption transition, We find clear evidence for the cross-over exponent to be smaller than $1 / 2$, in contrast to all previous simulations but in agreement with a re-summed field theoretic $\epsilon$-expansion. Since we use the pruned-enriched Rosenbluth method (PERM) which allows very precise estimates of the partition sum itself, we also obtain improved estimates for all entropic critical exponents.
\end{abstract}

\section{INTRODUCTION}

Single polymers grafted to a plane impenetrable wall have been studied intensely for a long time. If the surface is energetically neutral, the main effect is a change in the critical exponent governing the scaling of the partition sum. For an attractive surface the situation is more interesting, as there is a second order adsorption transition at a finite strength of the attraction 1].

Of particular interest is the cross-over exponent at this transition. Early simulations 1, 2] had given $\phi>0.5$, but it was claimed in [3] that this was due to finite size corrections to scaling, and that $\phi$ is actually very close to $1 / 2$ (the best estimated being $\phi=0.496 \pm 0.004$ ). Basically the same conclusion was reached recently in [4], where an even smaller error bar was obtained, $\phi=0.5005 \pm 0.0036$, suggesting that $\phi=1 / 2$ exactly. Since $\phi=1 / 2$ also in $d=2$ and $d \geq 4$, this would mean that $\phi$ is superuniversal, as it is for branched polymers [5, 6]. But a completely different picture was drawn in another recent paper [7], where it was claimed that $\phi=0.59$. To add to the confusion, we should cite field theoretic results. The $\epsilon$-expansion with $\epsilon=4-d$ predicts 8, 9] $\phi=1 / 2-\epsilon / 16+\left[16 \pi^{2}-39\right] \epsilon^{2} / 512+\ldots$. This would give $\phi=0.68$, if higher order terms were simply omitted, but Padé-Borel summation gives $\phi=0.483$ [10]. On the other hand, fixed dimension (massive theory) renormalization group calculations [10, 11] give $\phi=0.52$. All these calculations have errors which are hard to pin down, and the authors of [10] preferred the value 0.52 over 0.483 . Anyhow, these renormalization group calculations strongly suggest that $\phi$ is not superuniversal.

It is the purpose of the present work to clarify the situation by means of much more precise simulations. We will find that indeed $\phi$ is definitely smaller than $1 / 2$ (as predicted by the resummed $\epsilon$-expansion, but not by the supposedly more reliable massive field theory approach). In addition, we shall also provide precise estimates for the location of the adsorption transition and for the entropic exponents. The latter will be done both for thermal surfaces (at the adsorption transition point) and for a-thermal surfaces.

We model the polymer by a self avoiding walk (SAW) of $N-1$ steps on a simple cubic lattice with restriction $z \geq 0$. There is an energy $-\epsilon$ for each monomer (site) at $z=0$, the first monomer is located at $\mathbf{x}=0$. The temperature is taken to be $T=1$, so that the Boltzmann factor for each contact with the surface is $q=e^{\epsilon}$. The adsorption transition is at $q=q_{c}>1$. For the simulations we use the pruned-enriched Rosenbluth method (PERM) [12, 13]. This is a recursively (depth-first) implemented sequential sampling algorithm with re-sampling 14 . It is similar to the algorithm used in [3], but it is faster and much easier to use. Its main advantage over conventional Markov chain Monte Carlo (MC) methods is that it gives very precise estimates of the partition sum, without any need for thermodynamic integration or the like. To minimize statistical errors and speed up the algorithm, we use Markovian anticipation [15, 16]. We simulated $\approx 6.6 \times 10^{8}$ walks with $N=8000$ for $q=1$ and $\approx 9.5 \times 10^{8}$ walks at $q \approx q_{c}$. In both cases, about 1.6 $\%$ of these walks were strictly independent. Altogether this needed $\approx 5000$ hours CPU time on fast $(3 \mathrm{GHz})$ PCs. During the runs with $q>1$, results at slightly different values of $q$ were obtained by re-weighing on the fly, so that one run made with $q \approx q_{c}$ gave results at three close values of $q$. The critical point $q_{c}$ was then found by interpolation.

The partition sum is written as $Z_{1}(N, q)=$ $\sum_{m} C_{N m} q^{m}$, where $C_{N m}$ is the number of configurations with $m$ contacts with the wall, $z_{i_{k}}=0$ for $k=1, \ldots m$, and the subscript ' 1 ' indicates that one end is grafted. For $q<q_{c}$ it scales as

$$
Z_{1}(N, q) \sim \mu^{N} N^{\gamma_{1}-1}
$$

with $\mu$ and $\gamma_{1}$ independent of $q$, but with a $q$-dependent prefactor.

Near the adsorption transition, $Z_{1}(N, q)$ should scale as 1 .

$$
Z_{1}(N, q) \sim \mu^{N} N^{\gamma_{1}^{s}-1} \Psi\left[\left(q-q_{c}\right) N^{\phi}\right]
$$

where $\Psi(z)$ is analytic for finite $z$ and $\lim _{z \rightarrow-\infty} \Psi(z)$ is finite and positive. Notice that the dominant exponential growth of $Z_{1}(N, q)$ with $N$ is the same as for ordinary SAWs, as long as $q \leq q_{c}$. Taking the derivative of $\ln Z_{1}(N, q)$ with respect to $q$ and setting $q=q_{c}$ thereafter, we obtain for the average energy exactly at the 
critical point

$$
E_{N}\left(q_{c}\right)=\langle\epsilon m\rangle \sim N^{\phi} .
$$

Taking two derivatives we would obtain a scaling ansatz for the specific heat which is often used to estimate $\phi$ and other aspects of the critical behavior. We will not use it in the present paper, since Eqs.(2) and (3) give much more precise results, as already found in [3, 6]. We refer to [6] (which deals with the analogous problem for branched polymers) for a detailed discussion. Notice that Eq.(2) cannot be used with Markov chain MC methods, since the latter do not give simple and precise estimates of $Z_{1}(N, q)$ itself.

\section{RESULTS: A-THERMAL WALLS}

From previous simulations [3, 17] we know that $\mu \approx$ 4.68404, but the present simulations have higher statistics, therefore we should first estimate the critical exponent $\gamma_{1}$ in such a way that neither $\mu$ nor the unknown prefactor in Eq. (11) affect the value. For this we form the triple ratios 17]

$$
\gamma_{1, \mathrm{eff}}(N)=1+\frac{4 \ln Z_{1}(N)-3 \ln Z_{1}(N / 3)-\ln Z_{1}(3 N)}{\ln 9}
$$

(with $Z_{1}(N) \equiv Z_{1}(N, 1)$ ), which should tend to $\gamma_{1}$ as $N \rightarrow \infty$. The leading corrections to Eq. (11) (and thus also to Eq.(4) ) should scale as $1 / N^{\Delta}$ with the same exponent $\Delta \approx 0.5$ holding also for SAWs in absence of a surface 16 , 18, 19. This would suggest that we should get a straight line when plotting $\gamma_{1}(N)$ against $1 / \sqrt{N}$. Unfortunately this is not true, due to the presence of very large analytic corrections $\propto 1 / N, 1 / N^{2}, \ldots$. As seen from Fig. 1 , they shift the effective correction to scaling exponent to $\Delta_{\text {eff }} \approx$ 0.7 , and the extrapolation to $N \rightarrow \infty$ gives

$$
\gamma_{1}=0.6786 \pm 0.0012
$$

This is in good agreement with the best previous MC estimate $0.679 \pm 0.002[3]$ and with the field theoretic result 0.680 [10.

Using this value of $\gamma_{1}$, we next show in Fig. 2 the quantity $\ln Z_{1}(N)-a N+\left(1-\gamma_{1}\right) \ln N$ for different values of $a$, plotted again versus $1 / N^{\Delta}$. Again these data should extrapolate to $1 / N \rightarrow 0$ with a finite slope, if $a=\ln \mu$. From Fig. 2 we see that

$1 / \mu=0.21349098 \pm 0.00000005 \quad(\mu=4.6840386 \pm 0.0000011)$

where the error includes the uncertainty in the estimate of $\gamma_{1}$. This is the most precise value of the critical fugacity of SAWs on the simple cubic lattice published up to now. It is about 80 times more precise than the best estimate from exact enumerations [21].

In addition to the partition sum for SAWs grafted at one end to the surface, we can also study the partition

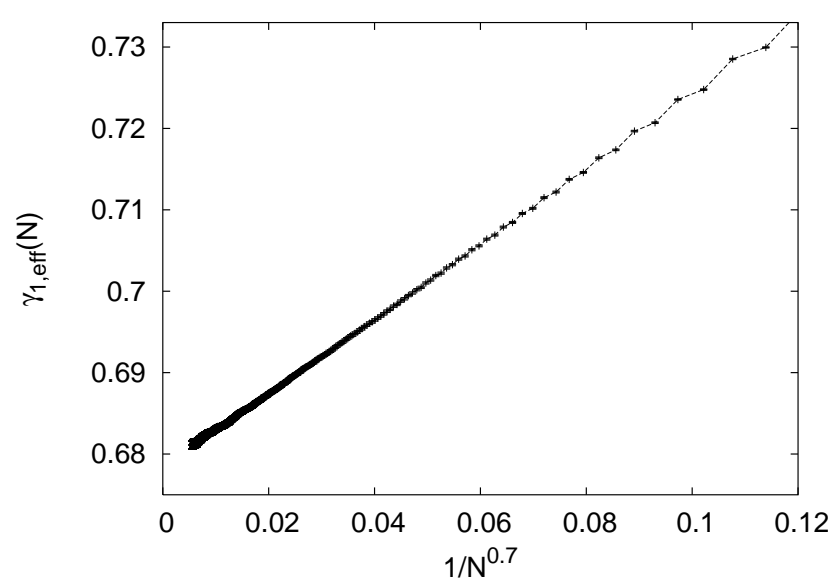

FIG. 1: Effective exponents defined by Eq. (4), plotted against $1 / N^{0.7}$.

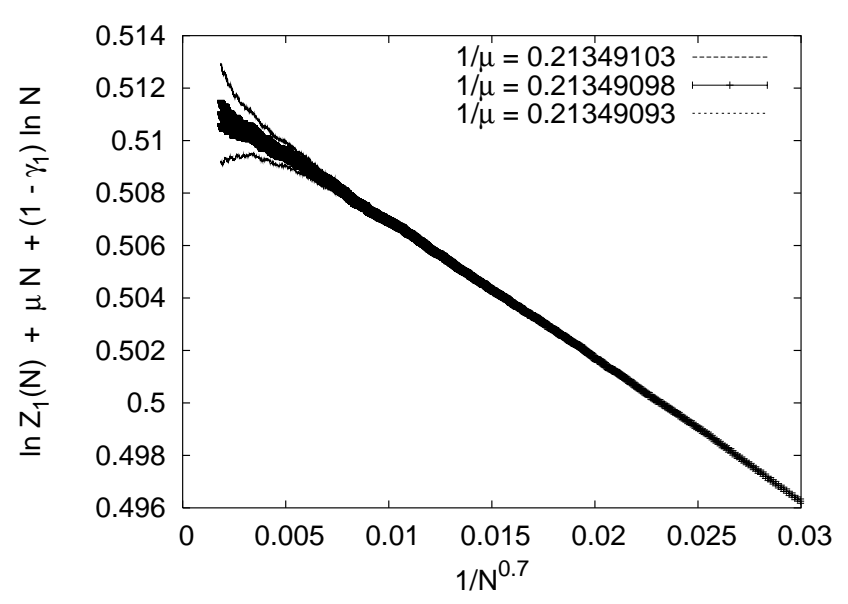

FIG. 2: The combination $\ln Z_{1}(N)-N \ln \mu+\left(1-\gamma_{1}\right) \ln N$, with $\gamma_{1}$ determined from Fig. 1 and for three candidate values of $\mu$, plotted against $1 / N^{0.7}$. Error bars are plotted only for the central curve, but errors are the same (within the precision of drawing) for all three curves.

sum $Z_{11}(N)$ where both ends are constrained to have $z=0$. In analogy to Eq.(10), it should scale as

$$
Z_{11}(N, q=1) \sim \mu^{N} N^{\gamma_{11}-1} .
$$

The exponent $\gamma_{11}$ should be related to previous exponents by the Barber relation [22]

$$
\gamma-2 \gamma_{1}+\gamma_{11}+\nu=0
$$

where $\gamma$ and $\nu$ are the entropy and Flory exponents $(R \sim$ $N^{\nu}$ ) for ungrafted SAWs in the bulk. Values of $Z_{11}(N)$ are obtained simply by summing over those walks for which the $N$-th monomer has $z=0$. Using the above estimate of $\mu$ and different candidate values of $\gamma_{11}$, we plot in Fig. 3 the analogous quantity to that shown in 


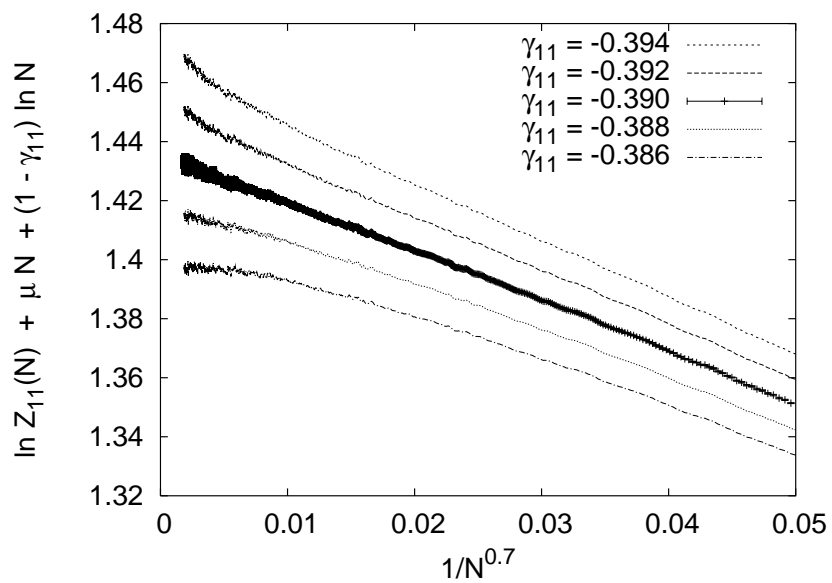

FIG. 3: Analogous to Fig. 2] but for SAWs grafted at both ends to the surface, and with the candidate values of $\mu$ replaced by candidate values of $\gamma_{11}$. In order to reduce statistical errors, the data are binned with bin width $\Delta N / N \approx 0.01$.

Fig. 2 for singly grafted chains. We find

$$
\gamma_{11}=-0.390 \pm 0.002,
$$

to be compared to the previous MC estimate $-0.383 \pm$ $0.005[3]$ and to the field theoretic prediction $-0.388[10]$. Using the most precise previous estimates of $\gamma$ and $\nu$ from [20] $(\gamma=1.1573 \pm 0.0002$ and $\nu=0.58765 \pm 0.00020)$, we see that the Barber relation is indeed perfectly satisfied,

$$
\gamma-2 \gamma_{1}+\gamma_{11}+\nu=-0.0023 \pm 0.0031
$$

Root mean square end-to-end distances should scale with the same Flory exponent as in the bulk, but with different prefactors and with different corrections to scaling. We measured both the components parallel and perpendicular to the wall for singly grafted SAWs. This time the corrections to scaling were $\sim 1 / \sqrt{N}$ as expected, i.e. there are much smaller analytic corrections. Results are shown in Fig. 4, where we divided averaged square distances by $N^{2 \nu}$ and plotted them against $1 / \sqrt{N}$. The ratio $\left\langle z_{N}^{2}\right\rangle /\left\langle x_{N}^{2}+y_{N}^{2}\right\rangle$ increases with $N$ as found also in [23], but it converges for $N \rightarrow \infty$ to a finite value, $0.938 \pm 0.002$. In contrast to 23 we see no indication that either $\left\langle z_{N}^{2}\right\rangle$ or $\left\langle x_{N}^{2}+y_{N}^{2}\right\rangle$ scales with an exponent different from the bulk Flory exponent.

\section{RESULTS: ATTRACTIVE WALLS}

According to Eq.(2), the exponential growth of the partition sum with $N$ is the same at $q_{c}$ as at $q=1$, i.e. we do not need the triple ratio Eq.(44) to estimate $\gamma_{1}^{s}(N)$. Instead we can use

$$
\gamma_{1, \mathrm{eff}}^{s}(N, q)=1+\frac{\ln \left[Z_{1}(2 N, q) / Z_{1}(N / 2, q) / \mu^{3 N / 2}\right]}{\ln 4}
$$

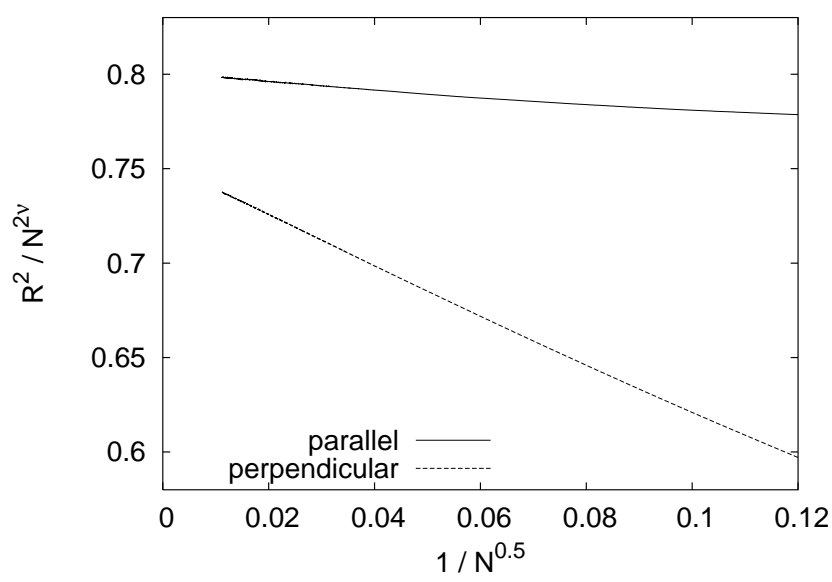

FIG. 4: $\left\langle x_{N}^{2}+y_{N}^{2}\right\rangle / N^{2 \nu}$ (upper curve) and $\left\langle z_{N}^{2}\right\rangle / N^{2 \nu}$ (lower curve) against $1 / \sqrt{N}$. Error bars are smaller than the thickness of the lines.

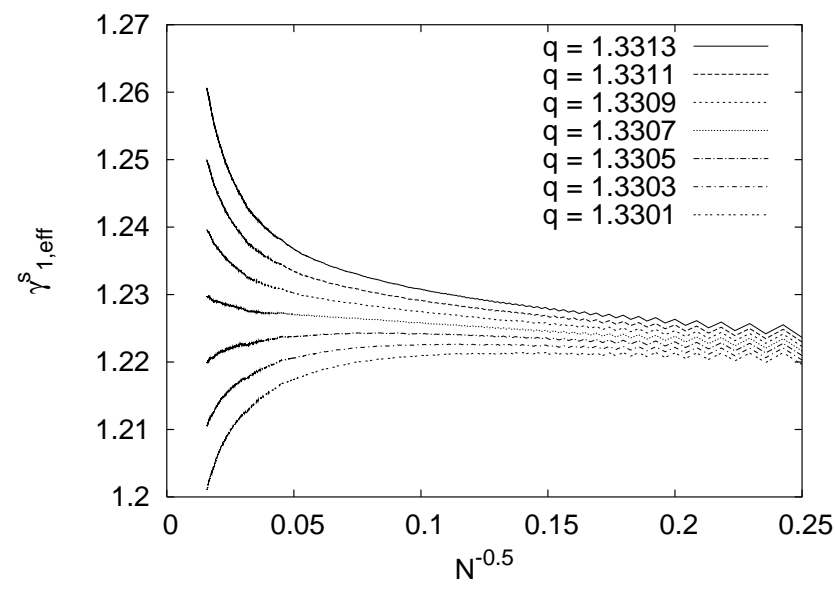

FIG. 5: Effective exponents $\gamma_{1, \text { eff }}^{s}(N, q)$, plotted versus $1 / \sqrt{N}$, for several values of $q$.

where the ratio between the two partition sums eliminates the unknown prefactor. The critical point is characterized by the fact that $\gamma_{1, \text { eff }}^{s}(N, q)$ diverges for $q>q_{c}$ when $N \rightarrow \infty$, converges slowly to $\gamma_{1}$ for $q<q_{c}$, and converges to a constant value larger than $\gamma_{1}$ exactly at $q_{c}$. Values of $\gamma_{1, \text { eff }}^{s}(N, q)$ obtained by means of Eq. (11) are shown in Fig. 5. As in the next figures to follow, we plotted it against $1 / \sqrt{N}$ since there was no different unique value of $\Delta$ which fitted all observables, and $\Delta=1 / 2$ was not worse overall than other values.

From Fig. 5 we see that $q_{c} \approx 1.3307$ and $\gamma_{1}^{s} \approx 1.23$, in good agreement with the estimates of [3]. More precise values will result by combining also the information from other observables.

The next quantity we looked at is the average energy, or rather the average number of sites in contact with the wall. Plotting simply $\langle m\rangle / N^{\phi}$ would suggest 


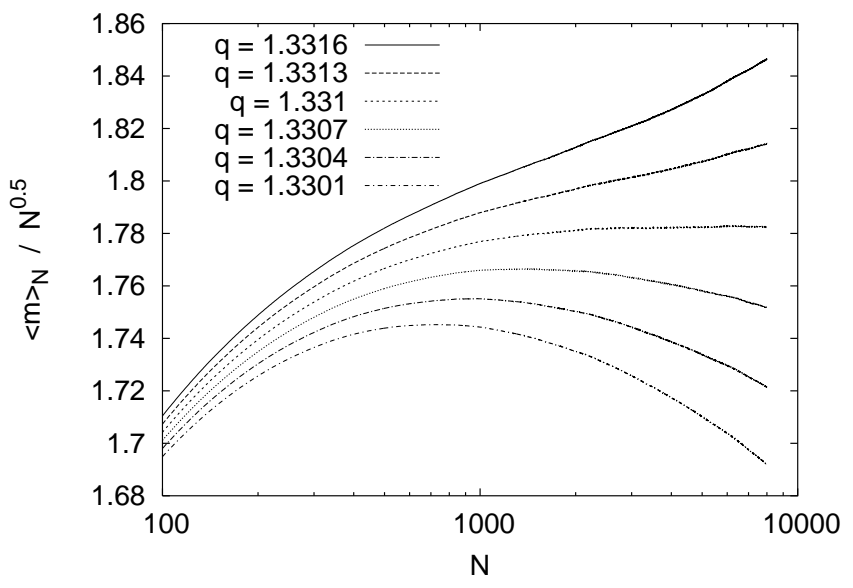

FIG. 6: Average number of contacts with the wall, divided by $\sqrt{N}$. This would suggest $\phi \approx 1 / 2$, if we would assume that corrections to scaling are negligible for $N>4000$.

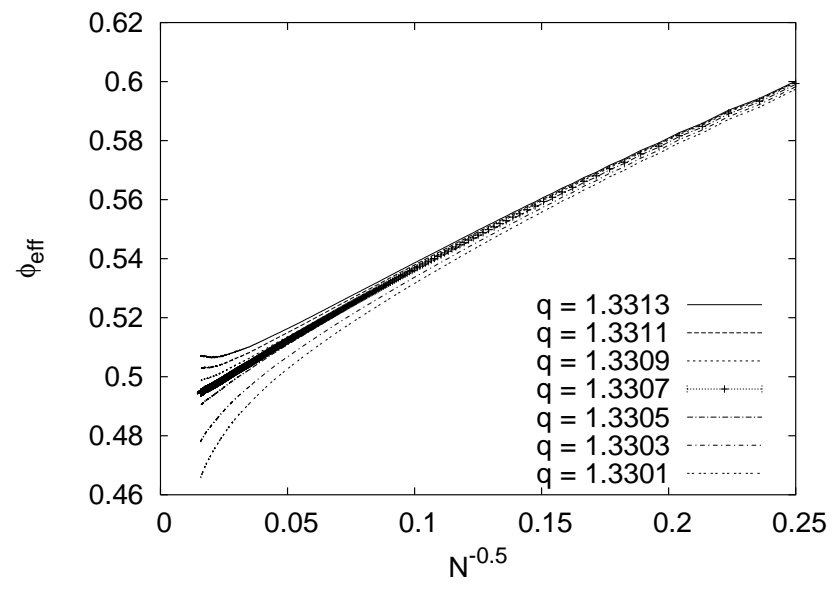

FIG. 7: Effective cross-over exponent as defined in the text, plotted versus $1 / \sqrt{N}$.

$\phi=1 / 2$ and $q_{c}=1.331$, see Fig. 6 . But this does not take into account the fact that corrections to scaling are very large and should still be important even for the largest $N$. Indeed, when defining an effective exponent by $\phi_{\text {eff }}(N, q)=(\ln 4)^{-1} \ln \left[E_{2 N}(q) / E_{N / 2}(q)\right]$ and plotting it against $1 / \sqrt{N}$ (Fig. 7), we see that it extrapolates clearly to a value $<1 / 2$, and that $q_{c}$ is closer to the value 1.3307 found from Fig. 5 .

Instead of the effective exponent $\gamma_{11, \text { eff }}^{s}(N, q)$ defined in complete analogy to Eq.(11), we show in Fig. 8 the difference $\gamma_{1, \text { eff }}^{s}(N, q)-\gamma_{11 \text {,eff }}^{s}(N, q)$. This is obtained from the ratio between the partition sums for singly and doubly grafted polymers, and is independent of the precise value of $\mu$. Finally, we show in Fig. 9 the ratio between the mean square end-to-end distances perpendicular and parallel to the wall.

Comparing Figs. 5 to 9, we see that all of them (except

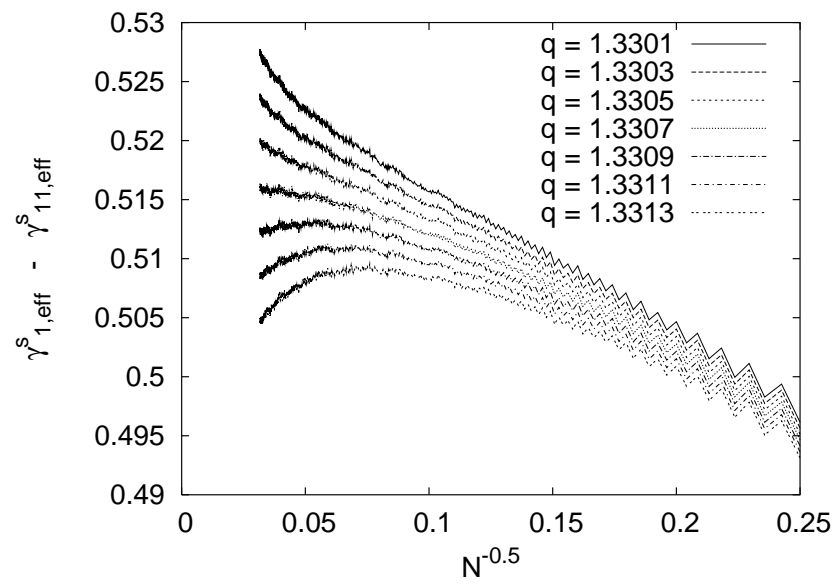

FIG. 8: Difference between the effective entropic exponent for polymers grafted at one end, and the exponent for polymers grafted at both ends, again plotted versus $1 / \sqrt{N}$.

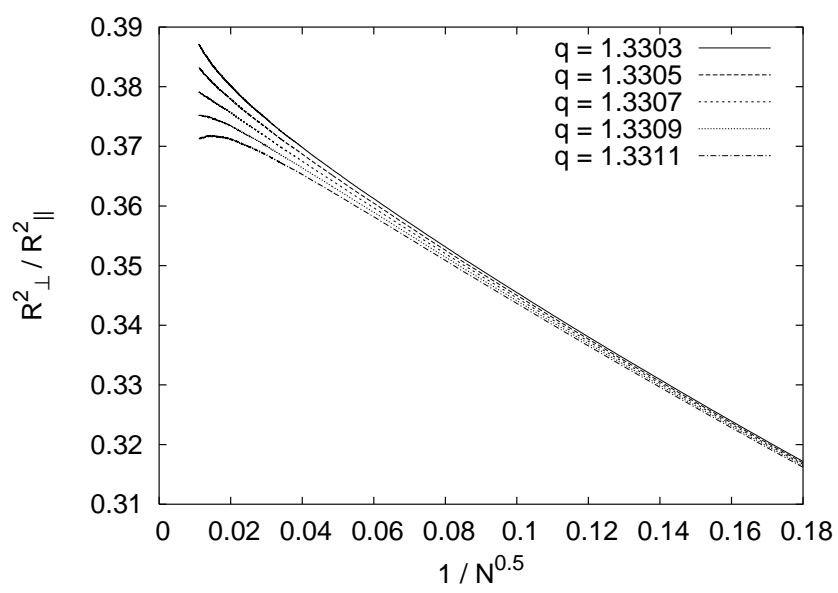

FIG. 9: Ratio $\left\langle z^{2}\right\rangle /\left\langle x^{2}+y^{2}\right\rangle$ between mean square end-to-end distances perpendicular and parallel to the wall.

of course Fig. 6) give the same value for $q_{c}$. Thus we obtain our precise estimate

$$
q_{c}=1.33065 \pm 0.00010
$$

This is in agreement with the value $1.3310 \pm 0.0003$ of [3], and with the recent estimate $1.334 \pm 0.026$ of [4]. For the anisotropy of infinitely long chains at the adsorption transition this gives $\left\langle z^{2}\right\rangle /\left\langle x^{2}+y^{2}\right\rangle=0.3845 \pm 0.0010$. The best estimates of the critical exponents are then

$$
\phi=0.484 \pm 0.002, \quad \gamma_{1}^{s}=1.226 \pm 0.002,
$$

and

$$
\gamma_{1}^{s}-\gamma_{11}^{s}=0.519 \pm 0.003
$$

These values are in less agreement with previous estimates. First of all, the entropic exponents are slightly 
smaller (by about 2 standard deviations) than the values given in 3], although these were already lower than all previous MC estimates. While $\gamma_{1}^{s}$ agrees roughly with the field theoretic prediction 1.207 of 10$], \gamma_{11}^{s}$ is quite a bit larger (0.707 as opposed to $\approx 0.666)$. Secondly, and more importantly, the cross-over exponent is now clearly less than $1 / 2$, by some 8 standard deviations. The closest previous MC estimate was $0.496 \pm 0.004$ [3], which is off by three sigma. The estimate $0.5005 \pm 0.0036$ of [4] seems clearly excluded by our data. It is based on very high statistics of very short $(N \leq 200)$ chains, which suggests that the corrections to scaling were not taken fully into account in [4]. As mentioned above, $\phi<1 / 2$ is predicted by the first order term of the $\epsilon$-expansion, but not when terms up to $O\left(\epsilon^{2}\right)$ are included and not by fixed dimension renormalization group methods. But the resummation of the $\epsilon$-expansion done in 10] gave $\phi=0.483$, in surprisingly good (and presumably fortuitous) agreement with our result. At least, our estimate is comfortably larger than the first order epsilon expansion result, $\phi>1 / 2-\epsilon / 16=0.4375$.

\section{CONCLUSION}

In this paper we have presented Monte Carlo simulations of single grafted 3 -d polymers in a good solvent, both for attractive and for a-thermal walls. The sample seems to be the biggest studied so far, both as concerns the number of chains simulated and their lengths. By using PERM which gives precise estimates of the partition sum, we could use the partition sum itself (instead of the specific heat) to locate the critical adsorption point and the critical exponents. Our estimates continue the decrease with increased statistics observed already in [3] . Our most interesting result is that the cross-over exponent is clearly less than $1 / 2$, in contrast to all previous simulations and to the best estimates from field theory.

I am indebted to Hsiao-Ping Hsu and Walter Nadler for discussions and for carefully reading the manuscript. I also thank Dieter W. Heermann for sending me Ref. 23. prior to publication.
[1] E. Eisenriegler, K. Kremer, and K. Binder, J. Chem. Phys. 77, 6296 (1982).

[2] H. Meirovitch and S. Livne, J. Chem. Phys. 88, 4507 (1988).

[3] R. Hegger and P. Grassberger, J. Phys. A: Math. Gen. 27, 4069 (1994).

[4] E.J. Janse van Rensburg and A.R. Rechnitzer, J. Phys. A: Math. Gen. 37, 6875 (2004).

[5] H. K. Janssen and A. Lyssy, Europhys. Lett. 29, 25 (1995).

[6] H.-P. Hsu, W. Nadler, and P. Grassberger, e-print cond-mat/0408061 (2004).

[7] R. Descas, J.-U. Sommer, and A. Blumen, J. Chem. Phys. 120, 8831 (2004).

[8] H.-W. Diehl and S. Dietrich, Phys. Rev. B 24, 2878 (1981).

[9] H.-W. Diehl, Phase Transitions and Critical Phenomena Vol. 10, C. Comb and J.L. Lebowitz (Eds.) (Academic Press, New York 1986).

[10] H.-W. Diehl and M. Shpot, Nucl. Phys. B 528, 595 (1998).

[11] H.-W. Diehl and M. Shpot, Phys. Rev. Lett. 73, 3431 (1994).

[12] P. Grassberger, Phys. Rev. E 56, 3682 (1997).

[13] P. Grassberger, Computer Physics Commun. 147, 64
(2002).

[14] J.S. Liu, Monte Carlo Strategies in Scientific Computing, Springer Series in Statistics (Springer, New York 2001).

[15] H. Frauenkron, P. Grassberger and N. Walter, e-print cond-mat/9806321 (1998).

[16] H.-P. Hsu and P. Grassberger, J. Chem. Phys. 120, 2034 (2004).

[17] P. Grassberger, P. Sutter, and L. Schäfer, J. Phys. A: Math. Gen. 30, 7039 (1997).

[18] B. Li, N. Madras, and A.D. Sokal, J. Statist. Phys. 80, 661 (1995).

[19] P. Belohorec and B.G. Nickel, Accurate Universal and Two-Parameter Model Results from a MonteCarlo Renormalization Group Study, Guelph University preprint (1997).

[20] H.-P. Hsu and P. Grassberger, Macromolecules 37, 4658 (2004).

[21] D. MacDonald, S. Joseph, D.L. Hunter, L.L. Moseley, N. Jan, and A. Guttmann, J. Phys. A: Math. Gen. 33, 5973 (2000).

[22] M.N. Barber, Phys. Rev. B 8, 407 (1973).

[23] J. Odenheimer and D.W. Heermann, Statistics of a Polymer Grafted to a Repulsive Wall, Univ. Heidelberg preprint (2004). 